\title{
Effective antioxidant, antimicrobial and anticancer activities of essential oils of horticultural aromatic crops in northern Egypt
}

\author{
Hosam O. Elansary ${ }^{1,2,3^{*}}$ (D, Samir A. M. Abdelgaleil ${ }^{4}$, Eman A. Mahmoud ${ }^{5}$, Kowiyou Yessoufou ${ }^{3}$, Khalid Elhindi ${ }^{1}$
} and Salah El-Hendawy'

\begin{abstract}
Background: Identifying ornamental plants as new natural antioxidant and antimicrobial sources is always of great importance for the ornamental and horticultural industries.

Methods: The antimicrobial activities of leaves and fruits peel essential oils of twelve ornamental and horticultural crops were determined by screening against wide spectrum of fungi and bacteria, and their respective in vitro antioxidant capacity was evaluated. Furthermore, the anticancer activities against several cancer cells, and one normal human cell line (HEK-293) were examined.

Results: Origanum vulgare L. essential oil showed the best antioxidant, antibacterial and anticancer activities compared to screened crops by means of the DPPH and linoleic acid assays for antioxidants, MIC and MBC values for antibacterial activities and $I_{50}$ for antiproliferative activities. Such important activities in $O$. vulgare was attributed to high pulegone ratio (77.45\%) as revealed by the GC/MS assay. Rosmarinus officinallis L. essential oil showed the highest antifungal activities by means of lowest MIC and MFC values which might be attributed to 1 , 8-cineole (19.60\%), camphor (17.01\%) and a-pinene (15.12\%).

Conclusion: We suggest that oxygenated monoterpenes (i.e. linalool, terpinen-4-ol and pulegone) and monoterpene hydrocarbons play an important role in the essential oil antioxidant, antibacterial, antifungal and anticancer activities of diverse Egyptian ornamental and horticultural crops. Some species showed bioactivities similar to standards compounds and might be suitable for pharmaceutical and food industries.
\end{abstract}

Keywords: Antioxidants, Ornamental plants, Essential oils, Chemical composition

\section{Background}

In an attempt to preserve human health and avoid food losses in the face of global growing food insecurity, synthetic preservatives were introduced in the food industries $[1,2]$. Unfortunately, some of these preservatives were reported to cause undesirable health side effects to humans $[1,2]$. For example, lipid peroxidation in stored food may lead to rancidity and reduction of food quality [3-5].

\footnotetext{
* Correspondence: helansary@ksu.edu.sa; hosammail2003@yahoo.com ${ }^{1}$ Plant production Department, College of Food and Agriculture Sciences, King Saud University, P.O. Box 2460, Riyadh 11451, Saudi Arabia

${ }^{2}$ Floriculture, Ornamental Horticulture and Garden Design Department, Faculty of Agriculture (El-Shatby), Alexandria University, Alexandria, Egypt Full list of author information is available at the end of the article
}

The consumption of spoiled food can cause a wide spectrum of human diseases. Furthermore, the development of resistant microorganisms to synthetic preservatives is another threat facing the continuous use of these chemicals.

Consequently, renewed interest has been placed on the discovery and use of natural bioactive resources in medicinal plants to control diseases, food spoilage microorganisms and oxidation [6-11]. Such medicinal plants include ornamental and horticultural crops that contain essential oils that are bioactive against the development of certain microorganisms [12-15]. The essential oils with high content of phenolics are recognized as strong

(c) The Author(s). 2018 Open Access This article is distributed under the terms of the Creative Commons Attribution 4.0 International License (http://creativecommons.org/licenses/by/4.0/), which permits unrestricted use, distribution, and reproduction in any medium, provided you give appropriate credit to the original author(s) and the source, provide a link to the Creative Commons license, and indicate if changes were made. The Creative Commons Public Domain Dedication waiver (http://creativecommons.org/publicdomain/zero/1.0/) applies to the data made available in this article, unless otherwise stated. 
antioxidants [16-20] and have antimicrobial activities against different types of microorganisms [21-27].

Essential oils might be used as anticancer agents as found before in Pinus koraiensis against HCT116 colorectal cancer cells [28], Myracrodruon urundeuva against HeLa, HEK-293, and Vero E6 cells [29] and Yemeni medicinal plants against A-427, 5637 and MCF-7 cancer cells [30]. Such activities might be attributed to major essential oil constitutes including cineol, capillin and others [31,32]. There are dozens of unstudied medicinal horticultural crops in Egypt with certain traditional uses such as skin, face and hair treatments [33] and urological, gastrointestinal, respiratory, neurological, cardiological and immunological diseases control [34]. This great diversity of alternative medicine application of essential oils and leaf extracts might be of pharmaceutical importance that need to be explored.

The study investigated the antioxidant and antimicrobial activities of essential oils obtained from important horticultural and economic ornamental medicinal plants. We also associate the main components of the essential oil with specific antioxidant and antimicrobial properties found to explore novel crop additive values.

\section{Methods}

\section{Plant materials}

The leaves or fruit peels of twelve plant species were collected from Alexandria, Behera and Matrouh in northern Egypt in the spring and summer of 2016. The leaves were obtained from mature non flowering herbal plants of Rosmarinus officinalis L., Artemisia judaica L., A. monosperma, Origanum vulgare L. and Pelargonium graveolens L'Her. Also the mature leaves of ornamental non trees of Rosmarinus officinalis Callistemon viminalis G. Don, Cupressus macrocarpa Gordon, Schinus molle L., and Thuja occidentalis L. The fruit peels were obtained from mature fruits of Citrus aurantifolia Swingle, C. limon (L.) and C. paradisi. The plant samples were collected during the months of April to August, 2016. Plants were identified by Prof. Samir Abdelgaleil and Assoc. Prof. Hosam Elansary. Specimens were vouchered in Department of Pesticide Chemistry and Technology, Alexandria University.

\section{Essential oils analysis}

The leaves were dried for five days $\left(26 \pm 1{ }^{\circ} \mathrm{C}\right)$ while the fruit peels were used fresh. The essential oils were obtained by hydrodistillation in a Clevenger-type apparatus for $1 \mathrm{~h}$. Gas chromatography/mass spectrometry (Hewlett Packard 5890) apparatus detected the the essential oil constitutes after dilution in diethyl ether. Gas chromatography column properties and injection conditions matches those described [3]. Oil compositions were identified by comparing retention time and indices of identified constitutes to n-alkanes $\left(\mathrm{C}_{10}-\mathrm{C}_{36}\right.$, Sigma-Aldrich, Cairo) subjected to the same conditions. NIST Ver. 2.0 and Wiley libraries were used as well for the identificaton of the compounds.

\section{Microorganisms and cell cultures}

The antimicrobial activities were investigated against fungi and bacteria. Fungi included Aspergillus flavus (ATCC 9643), A. ochraceus (ATCC 12066), A. niger (ATCC 6275), Penicillium ochrochloron (ATCC 48663), P. funiculosum (ATCC 56755), and Candida albicans (ATCC 12066)). Bacteria included Bacillus cereus (ATCC 14579), Micrococcus flavus (ATCC 10240), Listeria monocytogenes (clinical isolate), Staphylococcus aureus (ATCC 6538), Pseudomonas aeruginosa (ATCC 27853), Dickeya solani (DS 0432-1) and Escherichia coli (ATCC 35210),. The first four bacteria are Gram-positive while the last three are Gram-negative. The microorganisms are either of economic importance for the agricultural industry (e.g. L. monocytogenes, D. solani and S. aureus) or affect human health mainly such as $C$. albicans. Fungi and bacteria were obtained from Alexandria University. Cell cultures including breast adenocarcinoma (MCF-7), cervical adenocarcinoma (HeLa), T-cell lymphoblast (Jurkat), colon adenocarcinoma (HT-29) and urinary bladder carcinoma (T24) were purchased from American Type Culture Collection.

\section{Antioxidants}

The antioxidant activity of the essential oils was estimated using the DPPH [35] and the $\beta$-carotene-linoleic acid methods [35]. Positive control (BHT) and negative control were treated as samples and a calibration curve was obtained. The amount of the samples that inhibited $50 \%$ of each antioxidant solution $\left(\mathrm{IC}_{50}\right)$ were considered as the antioxidant activities and experiments were repeated twice.

\section{Antifungal activity assay}

The antifungal activities of the essential oils were estimated using the microdilution method [36]. The minimum inhibitory concentration (MIC) was the lowest concentration inhibiting the fungal growth using a binocular. The minimum fungicidal concentration (MFC) was estimated by serial sub-cultivations $(0.1-4.0 \mathrm{mg} /$ $\mathrm{mL}$ ). The MFC was determined as the concentration causing no visible growth and killing $99.5 \%$ of the original inoculum. Fluconazole (FLZ) and ketoconazole (KLZ) were used as positive control and experiments were repeated twice.

\section{Antibacterial activity assay}

The antibacterial activities were determined by the micro-dilution method [36]. To determine the minimum 
inhibitory (MIC) and minimum bactericidal (MBC) concentrations the MIC was considered as the lowest concentrations causing no growth using the binocular. The $\mathrm{MBC}$ was quantified using serial sub-cultivation of each bacterium $(0.1-2.0 \mathrm{mg} / \mathrm{mL})$ into $100 \mu \mathrm{L}$ of TSB and incubated for one day. MBC was the lowest concentration showed no growth and killed $99.5 \%$ of the original inoculum. Experiments were performed twice and negative control (5\% DMSO) as well as positive (streptomycin and ampicillin, $0.01-10 \mathrm{mg} / \mathrm{mL}$ ) were used.

\section{Antiproliferative activity assay}

Essential oils antiproliferative activities against MCF-7, HeLa, Jurkat, HT-29,T24 and HEK-293 followed the MTT method [37] with modifications. Five doses of leaf extracts were used to reach a final concentration of 50 , 100, 200, 300, and $400 \mu \mathrm{g} / \mathrm{mL}$ culture media. Negative controls and positive (vinblastine sulfate and taxol) were used.

Furthermore, $\mathrm{IC}_{50}$ values were obtained by plotting percentage of cell viability against extract concentration and expressed in $\mu \mathrm{g} / \mathrm{ml}$.

\section{Results}

\section{Essential oils GC-MS analyses}

The essential oils percentage and analyses (Table 1) showed that each species has its own chemical fingerprint. The major oil constitutes (\%) were $\beta$-thujone (49.83) and chrysanthenone (10.88) in A. judaica In $A$. monosperma, capillene (36.86) was the major compound while 1,8 -cineole (71.77) was the major in $C$. viminalis. The limonene (40.19) and $\beta$-pinene (19.65) were the major compounds in $C$. aurantifolia. In $C$. lemon, limonene (56.30) and $\beta$-pinene (8.81) were also the major compounds while in $C$. paradisi limonene (74.29) and linalool (4.61) were the main compounds. Terpinen-4-ol (20.29) and sabinene (18.67) were the major compounds in C. macrocarpawhile pulegone (77.45) and menthone (4.86) were majors in $O$. vulgare. $\beta$-citronellol (35.92) and geraniol (11.66) were major in $P$. graveolenswhile 1,8-cineole (19.60), camphor (17.01) and $\alpha$-pinene (15.12) were major in $R$. officinallis. $\alpha$-phellandrene (29.87) and $\beta$-phellandrene (21.08) were the major compounds in $S$. mollewhile $\alpha$-pinene (35.49) and $\delta$-3-carene (25.42) were the major in T. occidentalis.

The results of chemical analysis indicated that components including limonene, $\alpha$-pinene were found in more than one species, while others were species specific. Essential oils major constitutes could be divided into three groups including oxygenated monoterpenes (i.e. linalool and pulegone); monoterpene hydrocarbons (i.e. limonene and sabinene) and sesquiterpene hydrocarbons (i.e., $\sigma$-cadinene and $\sigma$-selinene).

\section{Antioxidant activities}

The essential oil of $O$. vulgare showed significantly the highest antioxidant activities with $\mathrm{IC}_{50}$ values of 2.8 and $1.1 \mathrm{mg} / \mathrm{L}$ in the DPPH and linoleic acid assays, respectively compared to other essential oils (Table 2). The oils of $A$. judaica, A. monosperma and C. viminalis followed $O$. vulgare oil in their antioxidant activities and their $\mathrm{IC}_{50}$ values ranged between $4.7-5.3$ and $2.7-3.3 \mathrm{mg} / \mathrm{L}$ in the DPPH and linoleic acid assays, respectively. The oil of S. molle showed the lowest antioxidant activity among all essential oils examined in the study. In addition, the oils of C. limon, C. macrocarpa, P. graveolens and $R$. officinallis showed no significant differences regarding their antioxidant activities.

\section{Antifungal activities}

The activities of the essential oils were expressed as MIC (Table 3) and MFC (Table 4). In general, all examined oils showed antifungal activities against A. flavus, $A$. ochraceus, A. niger, P. funiculosum, $P$. ochrochloron and C. albicans. The MIC and MFC values varied between $0.16-1.31$ and $0.33->4 \mathrm{mg} / \mathrm{mL}$, respectively. The oils of R. officinallis, O. vulgare, C. macrocarpa and C. aurantifolia showed the lowest MIC values compared to other plants species. The oil of $R$. officinallis showed the highest antifungal activities among studied essential oils by means of lowest values of MIC and MFC against the six fungi. The most resistant fungi were C. albicans, $P$. funiculosum and $A$. niger showing the highest MIC and MFC values. The essential oils of $R$. officinallis and $O$. vulgare revealed comparable activities to commercial reagents.

\section{The antibacterial activities}

There were large differences regarding essential oils antibacterial activities by means of MIC (Table 5) and MBC (Table 6). The oils of O. vulgare, C. macrocarpa and $C$. paradisi showed the highest antibacterial activities with MIC values ranged between $0.11-0.76 \mathrm{mg} / \mathrm{mL}$ against examined bacteria. O. vulgare, C. macrocarpa and C. paradise were the three most active essential oils, the $\mathrm{MBC}$ was in the range of 0.21 to 1.59 . The most resistant bacterium in this case of D. solani and L. monocytogenes. In general, the highest MIC values were recorded for the essential oils of A. monosperma, C. lemon, $R$. officinallis and S. molle. Most essential oils showed slightly higher MIC values compared to antibiotics, however, $O$. vulgare showed comparable values to antibiotics in some cases. The antibacterial activities were higher than antibiotics.

\section{Anticancer activities}

There were variations in essential oils anticancer activities against selected cancer cell lines as shown in Table 7. The inhibition (expressed as $\mathrm{IC}_{50}$ ) of different 
Table 1 Major constituents of essential oils extracted from twelve Egyptian plant species

\begin{tabular}{|c|c|}
\hline $\begin{array}{l}\text { Plant name } \\
\text { Oil yield (\% F.W., } v / W \text { ) }\end{array}$ & Major components $\left(\%, \mathrm{Rl}^{\mathrm{a}}\right)$ \\
\hline Artemisia judaica (0.2) & $\begin{array}{l}\beta \text {-Thujone }(49.83,1100), \text { Chrysanthenone }(10.88,1125) \text {, } \\
a \text { - Thujone (8.21,1116), 1,8-Cineole }(4.91,1034), \mathrm{L} \text {-Camphor } \\
(3.0,1192), \text { Artemisia alcohol }(2.20,1083)\end{array}$ \\
\hline Artemisia monosperma (0.8) & $\begin{array}{l}\text { Capillene }(36.86,1446) \text {, capillin }(14.68,1572), y \text {-Terpinene } \\
\text { (12.46,1047), } \beta \text {-Pinene }(7.85,964) \text {, cis-Ocimene }(3.26,1043) \text {, } \\
\text { Terpinen-4-ol }(2.59,1192)\end{array}$ \\
\hline Callistemon viminalis (0.5) & $\begin{array}{l}\text { 1,8-Cineole (71.77,1034), a-Pinene (11.47,946), Terpinen-4-ol } \\
\text { (3.18,1192), Octadecanoic acid (3.08,2172), 1-Phellandrene }(1.30,1054)\end{array}$ \\
\hline Citrus aurantifolia (0.75) & $\begin{array}{l}\text { Limonene }(40.19,1029), \beta \text {-Pinene }(19.65,964) \text {, a-Citral }(8.14,1240) \text {, } \\
\gamma \text {-Terpinene }(6.34,1047) \text {, a-Terpineol }(3.71,1185), \text { Terpinen-4-ol }(2.62,1192)\end{array}$ \\
\hline Citrus limon (0.2) & $\begin{array}{l}\text { Limonene }(56.30,1029), \beta \text {-Pinene }(8.81,964), \gamma \text {-Terpinene }(6.42,1047) \text {, } \\
\text { a-Citral }(4.96,1240), \beta \text {-Citral }(3.83,1216) \text {, a-Terpineol }(3.38,1185)\end{array}$ \\
\hline Citrus paradisi (0.12) & $\begin{array}{l}\text { Limonene }(74.29,1029) \text {, Linalool }(4.61,1117) \text {, Linalool oxide }(4.18,1088) \text {, } \\
\beta \text {-Citral }(2.66,1216) \text {, a-Fenchol }(1.99,1168) \text {, Nootkatone }(1.78,1800)\end{array}$ \\
\hline Cupressus macrocarpa (0.45) & $\begin{array}{l}\text { Terpinen-4-ol (20.29,1192), Sabinene (18.67,974), } \beta \text {-Citronellol } \\
\text { (13.01,1225), } \gamma \text {-Terpinene }(7.59,1047) \text {, Camphor }(6.66,1139), \\
\text { a-Terpinene }(4.50,1018)\end{array}$ \\
\hline Origanum vulgare (0.5) & $\begin{array}{l}\text { Pulegone }(77.45,1237) \text {, Menthone }(4.86,1152) \text {, cis-Isopulegone } \\
\text { (2.22, 1161), Piperitenone }(2.13,1253) \text {, Limonene }(1.08,1029) \text {, } \\
\text { Myrcene }(0.66,984)\end{array}$ \\
\hline Pelargonium graveolens (0.09) & $\begin{array}{l}\text { B-Citronellol (35.92,1225), Geraniol }(11.66,1233) \text {, Citronellylformate } \\
\text { (11.40,1275), Linalool }(9.63,1117),(+) \text {-Isomenthone }(6.36,1164) \text {, } \\
\sigma \text {-Selinene }(5.52,1484)\end{array}$ \\
\hline Rosmarinus officinalis (0.33) & $\begin{array}{l}\text { 1,8-Cineole (19.60,1034), Camphor (17.01,1139), a-Pinene } \\
\text { (15.12,946), Verbenone (9.55,1204), Borneol }(8.17,1188) \text {, } \\
\text { Linalool }(5.32,1117)\end{array}$ \\
\hline Schinus molle (0.88) & $\begin{array}{l}\text { a-Phellandrene (29.87,1005), } \beta \text {-Phellandrene }(21.08,1031) \text {, Elemol } \\
\text { (13.00,1547), T-Muurolol }(5.35,1641), \gamma \text {-Eudesmol }(4.48,1629) \text {, } \\
\sigma \text {-Cadinene }(3.99,1524)\end{array}$ \\
\hline Thuja occidentalis (0.25) & $\begin{array}{l}\text { a-Pinene }(35.49,946), \delta \text {-3-Carene }(25.42,1004), a \text {-Cedrol }(9.05,1585), \\
\text { a-Terpinolene }(6.76,1092) \text {, Limonene }(4.91,1029) \text {, Myrcene }(2.77,984)\end{array}$ \\
\hline
\end{tabular}

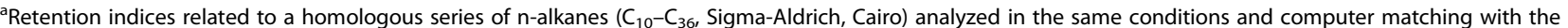
NIST mass spectral search program Ver. 2.0 and Wiley libraries

types of cancer cells proliferation ranged between 8.1 and $>300 \mu \mathrm{g} / \mathrm{ml}$. The highest antiproliferation activities were found in O. vulgare, Citrus sp. and A. monosperma against MCF-7, HeLa, Jurkat, HT-29 and T24 cancer cells. The lowest antiproliferative activities were found in the essential oils of P. graveolens. No inhibition activity was found in any oil against HEK-293 (kidney epithelial).

\section{Discussion}

Essential oils showed high variation in term of composition even among closely related species (i.e. species of the same genera), which is in agreement with previous reports on several plants [39-41]. All essential oils examined showed antioxidant capacities; however, $O$. vulgare showed the highest antioxidant activities. This might be attributed to specific essential oil such as the pulegone $(77.45 \%)$. The pulegone is a known compound found in the family Lamiaceae and has been associated with high antioxidant activities [42-44]. Although previous studies reported that pulegone is the major oil component of $O$. vulgare [44], environmental conditions of the plant may alter the chemical composition of the oil $[41,43]$ leading to the variation of chemical composition that we found among closely related species. Similarly, many other species showed high antioxidant activities (e.g. A. judaica, A. monosperma and C. viminalis). cajor components and their synergetic effects. $\beta \alpha$. Specifically, we found a high ratio of $\beta$-thujone (49.83\%) and $\alpha$-thujone $(8.21 \%)$ in A. Judaica, supporting the previous report in Artemisia sieberi Besser in Italy and Iran [45]. The essential oil ratio found in Italy and Iran [45] is slightly higher than that found in our study. However, A. monosperma showed high ratios of $\alpha$-pinene and terpinen-4-ol which are known to have noticeable antioxidant activities [46]. The high antioxidant activities that we found in relation with the presence of $\beta$ thujone may indicate that this compound may play a major role in the antioxidant activities of different Artemisia species. 
Table 2 Antioxidant activity of essential oils using the corresponding concentrations measured by DPPH and $\beta$-carotene-linoleic acid methods ${ }^{a}$

\begin{tabular}{|c|c|c|}
\hline \multirow[t]{2}{*}{ Essential oil } & \multicolumn{2}{|l|}{$\mathrm{IC}_{50}(\mathrm{mg} / \mathrm{L})$} \\
\hline & $\begin{array}{l}\text { DPPH radical } \\
\text { scavenging }\end{array}$ & $\begin{array}{l}\beta \text {-Carotene-linoleic } \\
\text { acid }\end{array}$ \\
\hline Artemisia judaica & $4.7 \pm 0.1 b$ & $2.7 \pm 0.1 b$ \\
\hline Artemisia monosperma & $5.3 \pm 0.3 c^{b}$ & $3.3 \pm 0.1 \mathrm{~cd}$ \\
\hline Callistemon viminalis & $5.2 \pm 0.1 \mathrm{C}$ & $3.2 \pm 0.1 \mathrm{c}$ \\
\hline Citrus aurantifolia & $7.2 \pm 0.3 e$ & $5.6 \pm 0.3 f$ \\
\hline Citrus limon & $6.1 \pm 0.3 d$ & $4.2 \pm 0.1 e$ \\
\hline Cupressus macrocarpa & $6.1 \pm 0.1 d$ & $4.2 \pm 0.3 e$ \\
\hline Origanum vulgare & $2.8 \pm 0.3 a$ & $1.1 \pm 0.1 \mathrm{a}$ \\
\hline Pelargonium graveolens & $6.2 \pm 0.1 d$ & $4.1 \pm 0.3 e$ \\
\hline Rosmarinus officinalis & $6.0 \pm 0.1 d$ & $4.2 \pm 0.2 e$ \\
\hline Schinus molle & $8.9 \pm 0.1 f$ & $6.6 \pm 0.3 \mathrm{~g}$ \\
\hline Thuja occidentalis & $5.6 \pm 0.3 \mathrm{~cd}$ & $3.6 \pm 0.4 d$ \\
\hline Butylated hydroxytoluene (BHT) & $2.9 \pm 0.1 \mathrm{a}$ & $2.6 \pm 0.2 b$ \\
\hline
\end{tabular}

${ }^{a}$ Values are expressed as means \pm SD

${ }^{\mathrm{b}}$ Means followed by different letters within a column indicate significant differences between treatments based on LSD test $(P \leq 0.05)$

The essential oil in $R$. officinallis showed the highest antifungal activities by means of lowest MIC and MFC values which might be attributed to 1,8-cineole (19.60\%), camphor $(17.01 \%)$ and $\alpha$-pinene $(15.12 \%)$ as the major oil constituents as previously reported [47]. It was reported that 1,8-cineole, terpinen-4-ol and other compounds showed strong in-vitro antifungal activities against some plant pathogenic fungi including Fusarium cerealis, Aspergillus tubingensis and A. carbonarius
[48]. In the current study, we report strong antifungal activities of the 1,8-cineole-rich $R$. officinallis against plant and human pathogenic fungi including A. flavus, A. ochraceus, A. niger, P. funiculosum, P. ochrochloron and $C$. albicans. The oil of $O$. vulgare followed $R$. officinallis in term of level of antifungal activities, and such interesting bioactivities of the essential oil might be attributed to the presence of pulegone $(77.45 \%)$ and the menthone (4.86\%).

The essential oil in C. macrocarpa showed high antifungal activities compared to other species, which is attributed to the presence of essential antifungal compounds including terpinen-4-ol (20.29\%), sabinene (18.67\%), $\gamma$-terpinene (7.59\%) and $\alpha$-terpinene (4.50\%) in agreement with previous investigations on other plants [46]. High ratios of limonene (40.19\%) and $\beta$-pinene (19.65\%) were found in C. aurantifolia, confirming previous report [40].

In the current study, we document the highest antibacterial activity for the $O$. vulgare essential oil by means of MIC and MBC values compared to other species, potentially because of the high pulegone ratio. Previous investigations reported strong antibacterial activities of essential oils of the pulegone-rich Mentha longifolia L. and pulegone-rich $O$. vulgare against different bacteria such as Pantoea agglumerans and human pathogenic Acinetobacter baumannii, respectively [43, 44]. The high antifungal as well as antibacterial activities of pulegone rich essential oils suggest that this compound might have a wide spectrum of antifungal and antibacterial activities. The oils of $C$. macrocarpa and $C$. paradise followed $O$. vulgare in their level of antibacterial activities, which might be attributed to terpinen-4-ol and

Table 3 Minimum inhibitory concentration (MIC) of essential oils on fungal strains

\begin{tabular}{|c|c|c|c|c|c|c|}
\hline \multirow[t]{2}{*}{ Essential oil } & \multicolumn{6}{|c|}{$\mathrm{MIC}(\mathrm{mg} / \mathrm{mL})$} \\
\hline & A. flavus & A. ochraceus & A. niger & P. funiculosum & P. ochrochloron & C. albicans \\
\hline Artemisia judaica & $0.31 \pm 0.01$ & $0.38 \pm 0.02$ & $0.31 \pm 0.01$ & $2.95 \pm 0.11$ & $0.41 \pm 0.03$ & $0.72 \pm 0.03$ \\
\hline Artemisia monosperma & $0.22 \pm 0.03$ & $0.29 \pm 0.01$ & $0.27 \pm 0.03$ & $0.71 \pm 0.03$ & $0.33 \pm 0.01$ & $0.72 \pm 0.11$ \\
\hline Callistemon viminals & $0.25 \pm 0.03$ & $0.43 \pm 0.01$ & $0.51 \pm 0.03$ & $0.96 \pm 0.07$ & $0.52 \pm 0.05$ & $0.63 \pm 0.03$ \\
\hline Citrus aurantifolia & $0.18 \pm 0.01$ & $0.19 \pm 0.01$ & $0.27 \pm 0.01$ & $1.31 \pm 0.11$ & $0.41 \pm 0.03$ & $0.35 \pm 0.01$ \\
\hline Citrus limon & $0.33 \pm 0.01$ & $0.29 \pm 0.01$ & $0.57 \pm 0.03$ & $3.55 \pm 0.21$ & $0.57 \pm 0.03$ & $0.39 \pm 0.03$ \\
\hline Citrus paradisi & $0.38 \pm 0.01$ & $0.52 \pm 0.03$ & $0.81 \pm 0.03$ & $1.35 \pm 0.09$ & $0.42 \pm 0.01$ & $0.81 \pm 0.05$ \\
\hline Cupressus macrocarpa & $0.17 \pm 0.01$ & $0.20 \pm 0.01$ & $0.26 \pm 0.02$ & $0.25 \pm 0.02$ & $0.28 \pm 0.01$ & $0.72 \pm 0.05$ \\
\hline Origanum vulgare & $0.16 \pm 0.01$ & $0.18 \pm 0.02$ & $0.22 \pm 0.01$ & $0.25 \pm 0.01$ & $0.33 \pm 0.01$ & $0.26 \pm 0.01$ \\
\hline Pelargonium graveolens & $0.23 \pm 0.01$ & $0.45 \pm 0.05$ & $0.39 \pm 0.01$ & $0.52 \pm 0.01$ & $0.21 \pm 0.01$ & $0.83 \pm 0.05$ \\
\hline Rosmarinus officinalis & $0.15 \pm 0.01$ & $0.27 \pm 0.01$ & $0.17 \pm 0.01$ & $0.23 \pm 0.01$ & $0.21 \pm 0.03$ & $0.22 \pm 0.01$ \\
\hline Schinus molle & $0.27 \pm 0.01$ & $0.31 \pm 0.01$ & $0.61 \pm 0.05$ & $0.43 \pm 0.01$ & $0.47 \pm 0.01$ & $0.97 \pm 0.09$ \\
\hline Thuja occidentalis & $0.27 \pm 0.01$ & $0.41 \pm 0.05$ & $0.45 \pm 0.03$ & $0.51 \pm 0.03$ & $0.19 \pm 0.01$ & $1.21 \pm 0.07$ \\
\hline Fuconazole & $0.14 \pm 0.01$ & $0.19 \pm 0.01$ & $0.16 \pm 0.01$ & $0.11 \pm 0.01$ & $0.20 \pm 0.01$ & $0.11 \pm 0.01$ \\
\hline Ketoconazole & $0.21 \pm 0.01$ & $0.20 \pm 0.01$ & $0.12 \pm 0.01$ & $2.00 \pm 0.09$ & $0.20 \pm 0.01$ & $0.21 \pm 0.01$ \\
\hline
\end{tabular}


Table 4 Minimum fungicidal concentration (MFC) of essential oils on fungal strains

\begin{tabular}{|c|c|c|c|c|c|c|}
\hline \multirow[t]{2}{*}{ Essential oil } & \multicolumn{6}{|c|}{ MFC (mg/mL) } \\
\hline & A. flavus & A. ochraceus & A. niger & P. funiculosum & P. ochrochloron & Candida albicans \\
\hline Artemisia judaica & $0.77 \pm 0.03$ & $0.63 \pm 0.03$ & $0.85 \pm 0.03$ & $>4$ & $0.95 \pm 0.05$ & $1.86 \pm 0.09$ \\
\hline Artemisia monosperma & $0.46 \pm 0.01$ & $0.61 \pm 0.02$ & $0.87 \pm 0.05$ & $1.81 \pm 0.10$ & $0.61 \pm 0.03$ & $1.63 \pm 0.13$ \\
\hline Callistemon viminalis & $0.63 \pm 0.05$ & $0.95 \pm 0.05$ & $1.13 \pm 0.08$ & $>2$ & $0.95 \pm 0.03$ & $1.1 \pm 0.05$ \\
\hline Citrus aurantifolia & $0.39 \pm 0.03$ & $0.41 \pm 0.02$ & $0.56 \pm 0.02$ & $>2$ & $0.83 \pm 0.03$ & $0.71 \pm 0.03$ \\
\hline Citrus limon & $0.74 \pm 0.05$ & $0.76 \pm 0.04$ & $1.43 \pm 0.07$ & $>4$ & $1.41 \pm 0.08$ & $0.91 \pm 0.05$ \\
\hline Citrus paradisi & $0.76 \pm 0.03$ & $>2$ & $>2$ & $3.91 \pm 0.17$ & $0.93 \pm 0.05$ & $1.59 \pm 0.07$ \\
\hline Cupressus macrocarpa & $0.37 \pm 0.03$ & $0.48 \pm 0.03$ & $0.49 \pm 0.03$ & $0.73 \pm 0.03$ & $0.68 \pm 0.03$ & $1.53 \pm 0.15$ \\
\hline Origanum vulgare & $0.35 \pm 0.01$ & $0.47 \pm 0.03$ & $0.47 \pm 0.03$ & $0.61 \pm 0.01$ & $0.71 \pm 0.02$ & $0.63 \pm 0.03$ \\
\hline Pelargonium graveolens & $0.47 \pm 0.01$ & $1.21 \pm 0.09$ & $0.83 \pm 0.01$ & $1.19 \pm 0.08$ & $0.49 \pm 0.03$ & $1.92 \pm 0.10$ \\
\hline Rosmarinus officinalis & $0.33 \pm 0.01$ & $0.59 \pm 0.03$ & $0.38 \pm 0.03$ & $0.57 \pm 0.03$ & $0.47 \pm 0.03$ & $0.49 \pm 0.05$ \\
\hline Schinus molle & $0.67 \pm 0.07$ & $0.67 \pm 0.07$ & $1.38 \pm 0.09$ & $1.12 \pm 0.06$ & $0.89 \pm 0.05$ & $2.3 \pm 0.11$ \\
\hline Thuja occidentalis & $0.63 \pm 0.03$ & $0.96 \pm 0.12$ & $1.75 \pm 0.11$ & $1.11 \pm 0.09$ & $0.52 \pm 0.02$ & $2.51 \pm 0.11$ \\
\hline Fuconazole & $0.20 \pm 0.03$ & $0.32 \pm 0.01$ & $0.27 \pm 0.01$ & $0.24 \pm 0.01$ & $0.31 \pm 0.01$ & $0.20 \pm 0.01$ \\
\hline Ketoconazole & $0.39 \pm 0.03$ & $0.40 \pm 0.01$ & $0.19 \pm 0.01$ & $3.75 \pm 0.07$ & $0.40 \pm 0.03$ & $0.41 \pm 0.03$ \\
\hline
\end{tabular}

sabinene in C. macrocarpa as well as limonene and linalool in C. paradise. A strong antibacterial activity of five different terpenoids was reported [49] against Campylobacter spp. In addition, it was previously reported that the monoterpene terpinen-4-ol enhanced the antibacterial performance of Melaleuca alternifolia essential oils against $S$. aureus and E. coli [50]. In the same trend, limonene is the major component of citrus essential oils in most species and was proven to have specific bacterial inactivation mechanism in E. coli and others [51] thus justifying the noticeable antibacterial activities of $C$. paradisi. However, other species studied here (C. limon and $C$. aurantifolia) showed slightly lower antibacterial activities which could be partially explained by lower ratios of limonene and synergetic effects with other oil constituents such as the linalool in C. paradisi. Some essential oils in $R$. officinallis and $O$. vulgare showed comparable antibacterial and antifungal activities to antibiotics in some cases, which may indicate that these essential oils might be useful sources of natural products

Table 5 Minimum inhibitory concentrations (MIC) of essential oils on bacterial strains

\begin{tabular}{|c|c|c|c|c|c|c|c|}
\hline \multirow[t]{2}{*}{ Essential oil } & \multicolumn{7}{|c|}{$\mathrm{MIC}(\mathrm{mg} / \mathrm{mL})$} \\
\hline & B. cereus & D. solani & E. coli & L. monocytogenes & M. flavus & P. aeruginosa & S. aureus \\
\hline Artemisia judaica & $0.35 \pm 0.01$ & $0.39 \pm 0.01$ & $0.45 \pm 0.01$ & $0.31 \pm 0.01$ & $0.23 \pm 0.03$ & $0.16 \pm 0.01$ & $0.27 \pm 0.01$ \\
\hline Artemisia monosperma & $0.43 \pm 0.01$ & $0.40 \pm 0.01$ & $0.58 \pm 0.03$ & $0.52 \pm 0.01$ & $0.31 \pm 0.01$ & $0.52 \pm 0.01$ & $0.38 \pm 0.00$ \\
\hline Callistemon viminals & $0.37 \pm 0.01$ & $0.31 \pm 0.01$ & $0.49 \pm 0.01$ & $0.33 \pm 0.01$ & $0.31 \pm 0.03$ & $0.39 \pm 0.01$ & $0.41 \pm 0.01$ \\
\hline Citrus aurantifolia & $0.23 \pm 0.01$ & $0.49 \pm 0.01$ & $0.21 \pm 0.01$ & $0.31 \pm 0.01$ & $0.27 \pm 0.03$ & $0.21 \pm 0.01$ & $0.25 \pm 0.01$ \\
\hline Citrus limon & $0.53 \pm 0.01$ & $0.49 \pm 0.01$ & $0.27 \pm 0.01$ & $0.43 \pm 0.01$ & $0.83 \pm 0.07$ & $0.21 \pm 0.01$ & $0.43 \pm 0.01$ \\
\hline Citrus paradisi & $0.15 \pm 0.01$ & $0.27 \pm 0.01$ & $0.21 \pm 0.01$ & $0.20 \pm 0.01$ & $0.13 \pm 0.03$ & $0.15 \pm 0.01$ & $0.17 \pm 0.01$ \\
\hline Cupressus macrocarpa & $0.19 \pm 0.01$ & $0.29 \pm 0.01$ & $0.26 \pm 0.01$ & $0.21 \pm 0.01$ & $0.13 \pm 0.03$ & $0.19 \pm 0.01$ & $0.24 \pm 0.01$ \\
\hline Origanum vulgare & $0.11 \pm 0.01$ & $0.76 \pm 0.01$ & $0.25 \pm 0.01$ & $0.40 \pm 0.01$ & $0.21 \pm 0.03$ & $0.15 \pm 0.01$ & $0.28 \pm 0.01$ \\
\hline Pelargonium graveolens & $0.25 \pm 0.01$ & $0.27 \pm 0.01$ & $0.19 \pm 0.01$ & $0.25 \pm 0.02$ & $0.15 \pm 0.01$ & $0.21 \pm 0.01$ & $0.20 \pm 0.01$ \\
\hline Rosmarinus officinalis & $0.40 \pm 0.01$ & $0.89 \pm 0.01$ & $0.43 \pm 0.01$ & $0.52 \pm 0.01$ & $0.25 \pm 0.03$ & $0.22 \pm 0.01$ & $0.69 \pm 0.01$ \\
\hline Schinus molle & $0.29 \pm 0.01$ & $0.63 \pm 0.01$ & $0.72 \pm 0.01$ & $0.71 \pm 0.03$ & $0.42 \pm 0.03$ & $0.51 \pm 0.01$ & $0.37 \pm 0.01$ \\
\hline Thuja occidentalis & $0.33 \pm 0.03$ & $0.41 \pm 0.03$ & $0.26 \pm 0.01$ & $0.31 \pm 0.01$ & $0.20 \pm 0.01$ & $0.41 \pm 0.01$ & $0.25 \pm 0.01$ \\
\hline Streptomycin & $0.07 \pm 0.01$ & $0.21 \pm 0.01$ & $0.09 \pm 0.01$ & $0.18 \pm 0.01$ & $0.9 \pm 0.01$ & $0.07 \pm 0.01$ & $0.21 \pm 0.01$ \\
\hline Ampicillin & $0.10 \pm 0.01$ & $0.30 \pm 0.01$ & $0.24 \pm 0.01$ & $0.18 \pm 0.01$ & $0.11 \pm 0.01$ & $0.12 \pm 0.01$ & $0.11 \pm 0.01$ \\
\hline
\end{tabular}


Table 6 Minimum bactericidal concentration (MBC) of essential oils on bacterial strains

\begin{tabular}{|c|c|c|c|c|c|c|c|}
\hline \multirow[t]{2}{*}{ Essential oil } & \multicolumn{7}{|c|}{$\mathrm{MBC}(\mathrm{mg} / \mathrm{mL})$} \\
\hline & B. cereus & D. solani & E. coli & L. monocytogenes & M. flavus & P. aeruginosa & S. aureus \\
\hline Artemisia judaica & $0.63 \pm 0.03$ & $1.13 \pm 0.01$ & $0.93 \pm 0.05$ & $0.72 \pm 0.01$ & $0.53 \pm 0.03$ & $0.31 \pm 0.03$ & $0.53 \pm 0.03$ \\
\hline Artemisia monosperma & $0.89 \pm 0.03$ & $0.84 \pm 0.05$ & $1.31 \pm 0.09$ & $1.23 \pm 0.09$ & $0.71 \pm 0.03$ & $1.96 \pm 0.01$ & $0.65 \pm 0.03$ \\
\hline Callistemon viminals & $0.67 \pm 0.01$ & $0.75 \pm 0.01$ & $0.91 \pm 0.07$ & $0.73 \pm 0.05$ & $0.76 \pm 0.01$ & $1.13 \pm 0.09$ & $0.96 \pm 0.03$ \\
\hline Citrus aurantifolia & $0.49 \pm 0.03$ & $0.81 \pm 0.03$ & $0.47 \pm 0.03$ & $0.63 \pm 0.07$ & $0.94 \pm 0.01$ & $0.61 \pm 0.01$ & $0.53 \pm 0.00$ \\
\hline Citrus limon & $1.31 \pm 0.01$ & $0.81 \pm 0.01$ & $0.49 \pm 0.03$ & $0.93 \pm 0.07$ & $>2$ & $0.40 \pm 0.03$ & $0.93 \pm 0.05$ \\
\hline Citrus paradisi & $0.37 \pm 0.01$ & $0.61 \pm 0.01$ & $0.43 \pm 0.03$ & $0.83 \pm 0.03$ & $0.25 \pm 0.01$ & $0.31 \pm 0.01$ & $0.63 \pm 0.03$ \\
\hline Cupressus macrocarpa & $0.38 \pm 0.01$ & $0.63 \pm 0.01$ & $0.51 \pm 0.03$ & $0.57 \pm 0.03$ & $0.27 \pm 0.01$ & $0.53 \pm 0.03$ & $0.47 \pm 0.03$ \\
\hline Origanum vulgare & $0.21 \pm 0.01$ & $1.59 \pm 0.01$ & $0.58 \pm 0.03$ & $0.83 \pm 0.03$ & $0.42 \pm 0.01$ & $0.34 \pm 0.03$ & $0.67 \pm 0.03$ \\
\hline Pelargonium graveolens & $0.59 \pm 0.03$ & $0.67 \pm 0.03$ & $0.83 \pm 0.03$ & $0.46 \pm 0.03$ & $0.33 \pm 0.01$ & $0.43 \pm 0.03$ & $0.51 \pm 0.03$ \\
\hline Rosmarinus officinalis & $0.91 \pm 0.01$ & $1.81 \pm 0.01$ & $0.82 \pm 0.03$ & $1.15 \pm 0.07$ & $0.71 \pm 0.01$ & $0.93 \pm 0.01$ & $1.73 \pm 0.03$ \\
\hline schinus molle & $0.83 \pm 0.01$ & $1.57 \pm 0.01$ & $1.78 \pm 0.03$ & $>2$ & $0.98 \pm 0.01$ & $1.13 \pm 0.01$ & $0.89 \pm 0.03$ \\
\hline Thuja occidentalis & $0.79 \pm 0.05$ & $0.83 \pm 0.01$ & $0.71 \pm 0.03$ & $0.97 \pm 0.05$ & $0.53 \pm 0.01$ & $0.94 \pm 0.01$ & $0.53 \pm 0.03$ \\
\hline Streptomycin & $0.14 \pm 0.01$ & $0.40 \pm 0.02$ & $0.40 \pm 0.01$ & $0.35 \pm 0.01$ & $0.19 \pm 0.01$ & $0.15 \pm 0.01$ & $0.41 \pm 0.03$ \\
\hline Ampicillin & $0.18 \pm 0.01$ & $0.55 \pm 0.01$ & $0.41 \pm 0.03$ & $0.30 \pm 0.01$ & $0.18 \pm 0.01$ & $0.25 \pm 0.01$ & $0.19 \pm 0.03$ \\
\hline
\end{tabular}

for commercial applications in the pharmaceutical and food industries.

Several studies indicated that Gram-positive bacteria are less resistant than negative ones against natural products such as essential oils [52]. This resistance had been attributed to the additional outer membrane (this is likely the case in our study) and that most resistant bacterium was D. solani. Finally, the essential oils compositions and percentages obtained from this study are comparable to studies on geranium [53], citrus [54], $C$. viminalis [55], R. officinallis [47], Artimisia [45], and $O$. vulgare [44]. However, the essential oils composition of the current collection of horticultural crops differed in some cases from those reported in the literature for $S$. molle (mainly composed of pinene [56], T. occidentalis (mainly composed of thujone and fenchone [57] and $O$. vulgare (mainly composed of pulegone). Interestingly, other report on S. molle found that the essential oil might be composed of cubenol (27.1\%) and caryophyllene oxide (15.3\%) [58]. Indeed, a great diversity is known in essential oil plants due to environmental as well as genetic factors.

Few species showed promising anticancer activities against the proliferation of cancer cells. The main effect of the essential oils is attributed to main constitutes such as the capillin in A. monosperama, limonene in the

Table 7 In vitro antiproliferative activity $\left[I C_{50}(\mu \mathrm{g} / \mathrm{ml})\right]$ of twelve aromatic plants essential oils on cancer cell lines

\begin{tabular}{|c|c|c|c|c|c|c|}
\hline & MCF-7 & HeLa & Jurkat & HT-29 & $\mathrm{T} 24$ & HEK-293 \\
\hline Artemisia judaica & $28.51 \pm 1.1$ & $54.13 \pm 1.5$ & $63.71 \pm 1.6$ & $73.01 \pm 2.1$ & $171.13 \pm 1.8$ & $>300$ \\
\hline Artemisia monosperma & $15.15 \pm 1.1$ & $9.1 \pm 0.1$ & $11.0 \pm 0.5$ & $10.1 \pm 0.5$ & $119.0 \pm 2.5$ & $>300$ \\
\hline Callistemon viminals & $25.15 \pm 0.3$ & $18.75 \pm 1.5$ & $53.10 \pm 3.5$ & $10.51 \pm 1.0$ & $166.15 \pm 2.8$ & $>300$ \\
\hline Citrus aurantifolia & $11.11 \pm 0.3$ & $58.75 \pm 1.5$ & $17.10 \pm 1.5$ & $230.84 \pm 4.1$ & $>300$ & $>300$ \\
\hline Citrus limon & $9.52 \pm 1.6$ & $51.04 \pm 1.2$ & $15.34 \pm 1.2$ & $231.91 \pm 5.1$ & $216.7 \pm 4.1$ & $>300$ \\
\hline Citrus paradisi & $8.1 \pm 1.5$ & $46.15 \pm 1.8$ & $14.52 \pm 1.9$ & $220 \pm 5.3$ & $113.6 \pm 5.1$ & $>400$ \\
\hline Cupressus macrocarpa & $25.4 \pm 2.6$ & $24.16 \pm 1.6$ & $30.54 \pm 3.4$ & $124.8 \pm 5.2$ & $>300$ & $>300$ \\
\hline Origanum vulgare & $8.11 \pm 1.0$ & $13.41 \pm 1.1$ & $27.05 \pm 2.1$ & $12.18 \pm 1.4$ & $105.5 \pm 2.3$ & $>300$ \\
\hline Pelargonium graveolens & $61.0 \pm 1.5$ & $51.24 \pm 3.1$ & $178.5 \pm 2.8$ & $195.33 \pm 5.4$ & $270.13 \pm 7.1$ & $>300$ \\
\hline Rosmarinus officinalis & $36.5 \pm 2.1$ & $27.25 \pm 1.5$ & $73.11 \pm 2.9$ & $18.17 \pm 2.0$ & $118.31 \pm 2.8$ & $>300$ \\
\hline Schinus molle & $41.33 \pm 2.1$ & $119.5 \pm 2.6$ & $14.85 \pm 1.7$ & $18.35 \pm 1.3$ & $>300$ & $>300$ \\
\hline Thuja occidentalis & $57.35 \pm 2.3$ & $22.5 \pm 1.7$ & $95.52 \pm 1.3$ & $125.5 \pm 3.9$ & $>300$ & $>300$ \\
\hline Vinblastine sulfate & - & $2.5 \pm 0.08$ & $0.1 \pm 0.05$ & $21.4 \pm 1.5$ & $63.31 \pm 1.7$ & $51.5 \pm 2.1$ \\
\hline Taxol & $0.08 \pm 0.005$ & - & - & - & - & - \\
\hline
\end{tabular}


Table 8 Common names and edible parts of twelve ornamental and horticultural Egyptian plant species

\begin{tabular}{|c|c|c|c|}
\hline Plant name & Common name & Edible/economic parts & Common uses \\
\hline Artemisia judaica & Judean wormwood & Leaves and flowers & Spice, soft drink and cosmetic uses [37] \\
\hline Artemisia monosperma & Delile & Leaves and flowers & Spice, soft drink and cosmetic uses [37] \\
\hline Callistemon viminalis & weeping bottlebrush & Leaves and flowers & $\begin{array}{l}\text { Ornamental and source of antioxidants, } \\
\text { antifungal and antibacterial products [47] }\end{array}$ \\
\hline Citrus aurantifolia & Key lime & Fruits and leaves & $\begin{array}{l}\text { The fruits are eaten and the essential oils } \\
\text { are extracted from the fruit coat. The leaves } \\
\text { are used for medicinal puposes }[46,58]\end{array}$ \\
\hline Citrus limon & lemon & Fruits and leaves & $\begin{array}{l}\text { The fruits are eaten and the essential oils } \\
\text { are extracted from the fruit coat. The leaves } \\
\text { are used for medicinal puposes }[46,58]\end{array}$ \\
\hline Citrus paradisi & grapefruit & Fruits and leaves & $\begin{array}{l}\text { The fruits are eaten and the essential oils } \\
\text { are extracted from the fruit coat. The leaves } \\
\text { are used for medicinal puposes }[46,58]\end{array}$ \\
\hline Cupressus macrocarpa & Monterey cypress & Stem, leaves and cones & $\begin{array}{l}\text { Ornamental and source of antioxidants, } \\
\text { antifungal and antibacterial products [38] }\end{array}$ \\
\hline Origanum vulgare & wild marjoram & Leaves and flowers & Spice, soft drink and cosmetic uses [25] \\
\hline Pelargonium graveolens & Geranium & Leaves and flowers & Spice, soft drink and cosmetic uses [45] \\
\hline Rosmarinus officinalis & Rosemary & Leaves and flowers & Spice, soft drink and cosmetic uses [25] \\
\hline Schinus molle & American pepper & Leaves, flowers and fruits & $\begin{array}{l}\text { Ornamental and source of antioxidants, } \\
\text { antifungal and antibacterial products [48] }\end{array}$ \\
\hline Thuja occidentalis & northern white-cedar & Stem, leaves and cones & $\begin{array}{l}\text { Ornamental and source of antioxidants, } \\
\text { antifungal and antibacterial products [49] }\end{array}$ \\
\hline
\end{tabular}

Citrus sp. and pulegone in O. vulgare. In agreement with our results in Artemisia judaica oil (mainly composed of thujone), Torres et al. [59] reported that the thujone enriched fraction has potential anticancer activities. The use of capillin (1-10 uM), isolated from A. monosperama, inhibited cell proliferation of HT29, MIA PaCa-2 and HEp-2 [32]. Several recent studies investigated the anticancer activities of several compounds. Murata et al. [31] found that 1,8-cineole (the main component of Callistemon viminals) suppress the prolifieration of colon cancer cells by inducing apoptosis (see Miller et al. [60] for in-depth review). On one hand, Shapira et al. [61] found that Terpinen-4-ol inhibited different cancer cells and Shehab and Abu-Gharbieh, [62] suggested that the essential oil of Micromeria Fruticosa L. (58.5\% pulegone) has antitumor activities against Human Colon Cancer and MCF7 with $\mathrm{IC}_{50}$ at 10 and $12.7 \mu \mathrm{g} / \mathrm{Ml}$, respectively. Fayed [63] on the other hand reported that geranium essential oil (29.90\% citronellol) had the highest anticancer activity with the $\mathrm{LC}_{50}$ values of $62.50 \mu \mathrm{g}$ / $\mathrm{ml}$ in NB4 cell line and $86.5 \mu \mathrm{g} / \mathrm{ml}$ in HL-60 cell line whereas Lin et al. [64] reported that $\alpha$-Phellandrene found in Schinus molle influenced cell cycle and apoptosis in murine leukemia WEHI-3 cells. Chen et al. $[65,66]$ found that $\alpha$-pinene has antitumor activities through inducing cell cycle arrest.

We aimed to highlight the biological activities in ornamental and horticultural aromatic plants against pathogenic microbes in the Mediterranean region. We demonstrated their antioxidant activities by two different methods and their promising role as natural antioxidant resources. One of the important factors influencing the decision of the farmer to grow specific horticultural crop is the availability of different ways of marketing such as the use of the whole crop as a fresh crop or the use of the essential oil for the pharmaceutical industries (Table 8 ). Here the bioactivity that we found for these crops is an added value that may encourage farmers to grow these crops and increase the marketing possibilities of their end crops. On the medical level, the pharmaceutical values of those medical crops are promising for pathogenic diseases and human cancer control.

\section{Conclusion}

O. vulgarehad the best antioxidant and antibacterial activities with a high and unique pulegone ratio (77.45\%). The essential oil of $R$. officinallis essential oil showed the highest antifungal activities by means of lowest MIC and MFC values which might be attributed to 1,8-cineole, camphor and $\alpha$-pinene. The essential oils of $O$. vulgare, Citrus sp. and $A$. monosperma showed the highest antiproliferation activities against different cancer cells. Oxygenated monoterpenes (i.e. linalool and pulegone) as well as monoterpene hydrocarbons including pinenes plays a pivotal role in the antioxidant, antibacterial, antifungal and anticancer activities of the essential oils of different horticultural aromatic plants. Some species showed antioxidant and antimicrobial activities comparable to 
standard compounds, which may indicate that these crops are valuable resources of natural compounds useful for pharmaceutical and food industries.

\section{Acknowledgments}

The authors would like to extend their sincere appreciation to the Deanship of Scientific Research at King Saud University, Saudi Arabia through research group No (RG 1436-020). The Faculty of Agriculture, Alexandria University (20162017) supported the current research.

\section{Funding}

The study was funded by the deanship of Scientific Research at King Saud University through research group No (RG 1436-020).

\section{Availability of data and materials}

The datasets supporting the conclusions of this article are included within the article

\section{Authors' contributions}

HOE, SAMA and EAM designed and performed the experiments. All authors contributed in validating, writing and approving the final version of the manuscript.

\section{Ethics approval and consent to participate}

The research does not involve human subjects or human materials.

\section{Consent for publication}

Not applicable.

\section{Competing interests}

The authors declare that they have no competing interests.

\section{Publisher's Note}

Springer Nature remains neutral with regard to jurisdictional claims in published maps and institutional affiliations.

\section{Author details}

${ }^{1}$ Plant production Department, College of Food and Agriculture Sciences, King Saud University, P.O. Box 2460, Riyadh 11451, Saudi Arabia. ${ }^{2}$ Floriculture, Ornamental Horticulture and Garden Design Department, Faculty of Agriculture (El-Shatby), Alexandria University, Alexandria, Egypt. ${ }^{3}$ Department of Geography, Environmental Management and Energy Studies, University of Johannesburg, Auckland Park Kingsway Campus (APK) campus 2006, South Africa. ${ }^{4}$ Department of Pesticide Chemistry and Technology, Faculty of Agriculture, Alexandria University, ElShatby, Alexandria 21545, Egypt. ${ }^{5}$ Department of Food Industries, Damietta University, Damietta, Egypt.

\section{Received: 20 September 2017 Accepted: 18 June 2018}

\section{Published online: 13 July 2018}

\section{References}

1. Misra G, Pavlostathis SG. Biodegradation kinetics of monoterpenes in liquid and soil- slurry systems. Appl Microbiol Biotechnol. 1997;47:572-7.

2. Tripathi P, Singh V, Naik S. Immune response to Leishmania: paradox rather than paradigm. FEMS Immunol Med Microbiol. 2007;51:229-42.

3. Mau JL, Huang PN, Huang SJ, Chen CC. Antioxidant properties of methanolic extracts from two kinds of Antrodia camphorate mycelia. Food Chem. 2004;86:25-31.

4. Dai J, Zhu L, Yang L, Qiu J. Chemical composition antioxidant and antimicrobial activities of essential oil from Wedelia prostrate. EXCLI J. 2013; 12:479-90.

5. Celiktas OY, Kocabas EEH, Bedir E, Verdar SO, Baser KHC. Antimicrobial activities of methanolic extract and essential oils of Rosmarinus officinalis, depending on location and seasonal variations. Food Chem. 2007:100:553-9.

6. Bajpai KV, Dung NT, Kwon JO, Kang SC. Analysis and the potential application of essential oil and leaf extracts of Silene Americana L. to control food spoilage and foodborne pathogens. J Food Technol. 2008;227:1613-20.

7. Raeisi S, Ojagh SM, Sharifi-Rad M, Sharifi-Rad J, Quek SY. Evaluation of Allium paradoxum (M.B.) G. Don. And Eryngium caucasicum trauve. Extracts on the shelf-life and quality of silver carp (Hypophthalmichthys molitrix) fillets during refrigerated storage. J Food Saf. 2017;37(3):e12321.

8. Sharifi-Rad J, Salehi B, Stojanović-Radić ZZ, Fokou PVT, Sharifi-Rad M Mahady GB, et al. Medicinal plants used in the treatment of tuberculosisethnobotanical and ethnopharmacological approaches. Biotechnol Adv 2017; S0734-9750(17)30077-0.

9. Sharifi-Rad M, Tayeboon GS, Sharifi-Rad J, Iriti M, Varoni EM, Razazi S. Inhibitory activity on type 2 diabetes and hypertension key-enzymes, and antioxidant capacity of Veronica persica phenolic-rich extracts. Cell Mol Biol (Noisy-Le-Grand). 2016;62:80-5.

10. Sharifi-Rad J, Salehi B, Varoni EM, Sharopov F, Yousaf Z, Ayatollahi SA, et al. Plants of the Melaleuca genus as antimicrobial agents: from farm to pharmacy. Phytother Res. 2017:31:1475-94.

11. Elansary HO, Szopa A, Kubica P, Ekiert H, Ali HM, Elshikh MS, et al. Bioactivities of traditional medicinal plants in Alexandria. Evid-Based Complementary Altern Med. 2018;2018:1463579.

12. Politeo O, Jukic M, Milos M. Chemical composition and antioxidant activity of essential oils of twelve spice plants. Croat Chem Acta. 2006; 79:545-52.

13. Adrar N, Oukil N, Bedjou F. Antioxidant and antibacterial activities of Thymus numidicus and Salvia officinalis essential oils alone or in combination. Ind Crop Prod. 2016;88:112-9.

14. Si Said ZB, Haddadi-Guemghar H, Boulekbache-Makhlouf L, Rigou P, Remini H, Adjaoud A, Khoudja NK, Madani K. Essential oil composition, antibacterial and antioxidant activities of hydrodistillated extract of Eucalyptus globulus fruits. Ind Crop and Prod. 2016;89:167-75.

15. Elansary HO, Yessoufou K, Shokralla S, Mahmoud EA, Skalicka-Woźniak K. Enhancing mint and basil oil composition and antibacterial activity using seaweed extracts. Ind Crop Prod. 2016;92:50-6.

16. Sharifi-Rad J, Sureda A, Tenore GC, Daglia M, Sharifi-Rad M, Valussi M, et al. Biological activities of essential oils: from plant Chemoecology to traditional healing systems. Molecules. 2017:22:70.

17. Salem MZM, Elansary HO, Ali HM, El-Settawy AA, Elshikh MS, Abdel-Salam EM, Skalicka-Wozniak K. Bioactivity of essential oils extracted from Cupressus macrocarpa branchlets and Corymbia citriodora leaves grown in Egypt. BMC Complement Altern Med. 2018:18:23.

18. Sermukhamedova O, Wojtanowski KK, Widelski J, Korona-Głowniak I, Elansary HO, Sakipova Z, Malm A, Skalicka-Woźniak K. Metabolic profile of and antimicrobial activity in the aerial part of Leonurus turkestanicus V.I. Krecz. et Kuprian. from Kazakhstan. J AOAC Int. 2017;100:1700-5.

19. Radonic A, Milos M. Chemical composition and in vitro evaluation of antioxidant effect of free volatile compounds from Satureja montana L. Free Radic Res. 2003:37:673-9.

20. Kulisic T, Radonic A, Katalinic V, Milos M. Use of different methods for testing antioxidative activity of oregano essential oil. Food Chem. 2004;85:633-40.

21. Jerbi A, Zehri S, Abdnnabi R, Gharsallah N, Kammoun M. Essential oil composition, free-radical-scavenging and antibacterial effect from stems of Ephedra alata alenda in Tunisia. J Essent Oil Bear Plants. 2016;19: 1503-9.

22. Bhat G, Rassol S, Rehman S, Ganaie M, Qazi PH, Shawl AS. Seasonal variation in chemical composition, antibacterial and antioxidant activities of the essential oil of leaves of Salvia officinalis (sage) from Kashmir, India. J Essent Oil Bearing Plant. 2016:19:1129-40.

23. Sartoratto A, Machado ALM, Delarmelina C, Figueira GM, Dusrte MCT, Rehder VLG. Composition and antimicrobial activity of essential oils from aromatic plants used in Brazil. Braz J Microb. 2004;35:275-80.

24. Sonboli A, Eftekhar F, Yousefzadi M, Kanani MR. Antibacterial activity and chemical composition of the essential oil of Grammosciadium platycarpum Boiss. From Iran. Z Naturforsch. 2005;60:30-4.

25. Soković M, Marin PD, Brkić D, Griensven L. Chemical composition and antibacterial activity of essential oils of ten aromatic plants against human pathogenic bacteria. Food. 2007:1:220-6.

26. Celikel N, Kavas G. Antimicrobial properties of some essential oils against some pathogenic microorganisms. Czech J Food Sci. 2008:26:174-81.

27. Inaid Y, Yacoub R, Al-Biski F. Antioxidant and antimicrobial activities of Origanum vulgare essential oil. Int Food Res J. 2016:23:1706-10.

28. Cho S, Lee E, Kim S, Lee H. Essential oil of Pinus koraiensis inhibits cell proliferation and migration via inhibition of p21-activated kinase 1 pathway in HCT116 colorectal cancer cells. BMC. BMC Complement Altern Med. 2014;14:275.

29. Rebouças de Araújo ID, Coriolano de Aquino N, Guerra ACV, Júnior RF, Araújo RM, Júnior RF, Farias KJS, Fernandes JV, Andrade SV. Chemical 
composition and evaluation of the antibacterial and cytotoxic activities of the essential oil from the leaves of Myracrodruon urundeuva. BMC Complement Altern Med. 2017;17:419.

30. Mothana RA, Lindequist U, Gruenert R, Bednarski PJ. Studies of the in vitro anticancer, antimicrobial and antioxidant potentials of selected Yemeni medicinal plants from the island Soqotra. BMC Complement Altern Med. 2009;9:7.

31. Murata S, Shiragami R, Kosugi C, Tezuka T, Yamazaki M, Hirano A, Yoshimura Y, Suzuki M, Shuto K, Ohkohchi N, Koda K. Antitumor effect of 1, 8-cineole against colon cancer. Oncol Rep. 2013;30(6):2647-52.

32. Lee G, Park H-G, Choi M-L, Kim YH, Park YB, Song K-S, Cheong C, Bae Y-S. Falcarindiol, a Polyacetylenic compound isolated from Peucedanum japonicum, Inhibits Mammalian DNA Topoisomerase I. J Microbiol Biotechnol. 2000;10:394-8.

33. Elansary HO, Mahmoud EA, Shokralla S, Yessoufou K. Diversity of plants, traditional knowledge and practices in local cosmetics: a case study from Alexandria, Egypt. Econ Bot. 2015;69:114-26.

34. AbouZid SF, Mohamed AA. Survey on medicinal plants and spices used in Beni-Sueif, upper Egypt. J Ethnobiol Ethnomed. 2011;7:18.

35. Elansary HO, Mahmoud EA. Basil cultivar identification using chemotyping still favored over genotyping using core barcodes and possible resources of antioxidants. J Essent Oil Res. 2015;27:82-7.

36. Espinel-Ingroff A. Comparison of the E-test with the NCCLS M38-P method for antifungal susceptibility testing of common and emerging pathogenic filamentous fungi. J Clini Microbiol. 2001;39:1360-7.

37. Mosmann T. Rapid colorimetric assay for cellular growth and survival: application to proliferation and cytotoxicity assays. Immunol Methods. 1983;65:55-63.

38. Parry J, Su L, Moore J, Cheng Z, Luther M, Rao JN, Wang J-Y, Yu LL. Antiproliferative activities of selected fruit seed flours. J Agric Food Chem. 2006;54:3773-8.

39. Crockett SL. Essential oil and volatile components of the genus Hypericum (Hypericaceae). Nat Prod Commun. 2010;5:1493-506.

40. Sharma A, Cannoo DS. Phytochemical composition of essential oils isolated from different species of genus Nepeta of Labiatae family: a review. Pharmacophore. 2013;4:181-211.

41. Elansary HO. Basil morphological and physiological performance under trinexapac-ethyl foliar sprays and prolonged irrigation intervals. Acta Physiol Plant. 2015;37:1-13.

42. Mkaddem M, Bouajila J, Ennajar M, Lebrhi A, Mathieu F, Romdhane M. Chemical composition and antimicrobial and antioxidant activities of Mentha (longifolia L. and viridis) essential oils. J Food Sci. 2009;74:358-63.

43. Elansary HO, Ashmawy NA. Essential oils of mint between benefits and hazards. J Essent Oil Bearing Plant. 2013;16:429-38.

44. Saghi H, Bahador A, Khaledi A, Kachoei RA, Dastjerdi AF, Esmaeili D. Antibacterial effects of Origanum vulgare essence against multidrugresistant Acinetobacter baumannii isolated from selected hospitals of Tehran. Iran Avicenna J Clin Microb Infec. 2015;2:e22982.

45. Mahboubi M, Valian M, Kazempour N. Chemical composition, antioxidant and antimicrobial activity of Artemisia sieberi oils from different parts of Iran and France. J Essent Oil Res. 2015;27:140-7.

46. Elansary HO, Salem MZM, Ashmawy NA, Yacout M. Chemical composition, antimicrobial and antioxidant activities of Lantana camara, Cupressus sempervirens and Syzygium cumini leaves oils from Egypt. J Agric Sci. 2012; 4:144-52.

47. Takayama C, de-Faria FM, de Almeida ACA, Dunder RJ, Manzo LP, Socca EAR, Batista LM, Salvador MJ, Souza-Brito ARM, Luiz-Ferreira A. Chemical composition of Rosmarinus officinalis essential oil and antioxidant action against gastric damage induced by absolute ethanol in the rat. Asian Pac J Trop Biomed. 2016;6:677-81.

48. Morcia C, Malnati M, Terzi V. In vitro antifungal activity of terpinen-4-ol, eugenol, carvone, 1,8-cineole (eucalyptol) and thymol against mycotoxigenic plant pathogens. Food Addit Contam Part A Chem Anal Control Expo Risk Assess. 2012;29:415-22.

49. Kurekci C, Padmanabha J, Bishop-Hurley SL, Hassan E, Al Jassim RA, McSweeney CS. Antimicrobial activity of essential oils and five terpenoid compounds against Campylobacter jejuni in pure and mixed culture experiments. Int J Food Microbiol. 2013;166:450-7.

50. Hammer KA, Carson CF, Riley TV. Effects of Melaleuca alternifolia (tea tree) essential oil and the major monoterpene component terpinen-4-ol on the development of single- and multistep antibiotic resistance and antimicrobial susceptibility. Antimicrob Agents Chemother. 2012;56:909-15.
51. Espina L, Gelaw TK, de Lamo-Castellví S, Pagán R, García-Gonzalo D. Mechanism of bacterial inactivation by (+)-limonene and its potential use in food preservation combined processes. PLoS One. 2013;8:e56769.

52. Aelenei P, Miron A, Trifan A, Bujor A, Gille E, Aprotosoaie AC. Essential oils and their components as modulators of antibiotic activity against gramnegative bacteria. Medicines. 2016:3:1-34.

53. Sharopov FS, Zhang H, Setzer WN. Composition of geranium (Pelargonium graveolens) essential oil from Tajikistan. American J Essen Oils Nat Prod. 2014;2:13-6.

54. Dejnane D. Chemical profile, antibacterial and antioxidant activity of Algerian citrus essential oils and their application in Sardina pilchardus. Foods. 2015:4:208-28.

55. Salem MZM, El-Hefny M, Nasser R, Ali HM, El-Shanhorey NA, Elansary HO. Medicinal and biological values of Callistemon viminalis extracts: history, current situation and prospects. Asian Pacific J Trop Med. 2017:10:229-37.

56. Diaz C, Quesada S, Brenes O, Aguilar G, Ciccio JF. Chemical composition of Schinus molle essential oil and its cytotoxic activity on tumour cell lines. Nat Prod Res. 2008;22:1521-34.

57. Svajdlenka E, Mártonfi P, Tomasko I, Grancai D, Nagy M. Essential oil compostion of Thuja occidentalis L. samples from Slovakia. J Essn Oil Res. 1998;11:532-6.

58. Cavalcanti AS, Alves MS, Paulo da Silva LC, Sanches MN, Chaves DSA, Alves de Souza MA. Volatiles composition and extraction kinetics from Schinus terebinthifolius and Schinus molle leaves and fruit. Rev Bras Farm. 2015;25: 356-62.

59. Torres A, Vargas Y, Uribe D, Carrasco C, Torres C, Rocha R, Oyarzún C, San Martín R, Quezada C. Pro-apoptotic and anti-angiogenic properties of the a / $\beta$-thujone fraction from Thuja occidentalis on glioblastoma cells. J NeuroOncol. 2016;128:9-19.

60. Miller JA, Thompson PA, Hakim IA, Chow HS, Thomson CA. D-limonene: a bioactive food component from citrus and evidence for a potential role in breast cancer prevention and treatment. Oncol Rev. 2011;5:31-42.

61. Shapira S, Pleban S, Kazanov D, Tirosh P, Arber N. Terpinen-4-ol: a novel and promising therapeutic agent for human gastrointestinal cancers. PLoS One. 2016;11:e0156540.

62. Shehab NG, Abu-Gharbieh E. Constituents and biological activity of the essential oil and the aqueous extract of Micromeria fruticosa (L.) Druce subsp. serpyllifolia. Pak J Pharm Sci. 2012;25:687-92.

63. Fayed SA. Antioxidant and anticancer activities of Citrus reticulate (Petitgrain mandarin) and Pelargonium graveolens (Geranium) essential oils. Res J Agri Biol Sci. 2009:5:740-7.

64. Lin JJ, Yu CC, Lu KW, Chang SJ, Yu FS, Liao CL, Lin JG. Chung JG.aPhellandrene alters expression of genes associated with DNA damage, cell cycle, and apoptosis in murine leukemia WEHI-3 cells. Anticancer Res. 2014; 34:4161-80

65. Chen W, Liu Y, Li M, Mao J, Zhang L, Huang R, Jin X, Ye L. Anti-tumor effect of a-pinene on human hepatoma cell lines through inducing G2/M cell cycle arrest. J Pharmacol Sci. 2015;127:332-8.

66. Bourgou S, Rahali FZ, Ourghemmi I, Tounsi S. Changes of peel essential oil composition of four Tunisian Citrus during fruit maturation. Scientific World J. 2012;2012:528593

\section{Ready to submit your research? Choose BMC and benefit from:}

- fast, convenient online submission

- thorough peer review by experienced researchers in your field

- rapid publication on acceptance

- support for research data, including large and complex data types

- gold Open Access which fosters wider collaboration and increased citations

- maximum visibility for your research: over $100 \mathrm{M}$ website views per year

At BMC, research is always in progress.

Learn more biomedcentral.com/submissions 